\title{
The risks, degree of malignancy and clinical progression of prostate cancer associated with the MDM2 T309G polymorphism: a meta-analysis
}

\author{
Jie Yang ${ }^{1, *}$, Wen Gao ${ }^{2, *}$, Ning-Hong Song ${ }^{1}$, Wei Wang ${ }^{1}$, Jie-Xiu Zhang ${ }^{1}$, Pei Lu ${ }^{1}$, Li-Xin Hua ${ }^{1}$ and Min Gu ${ }^{1}$
}

To determine the risk, malignant degree and clinical progression of prostate cancer ( $\mathrm{PCa}$ ) associated with mouse double-minute 2 protein (MDM2) T309G variants, a meta-analysis was performed on all eligible published studies. Odds ratios (ORs) with $95 \%$ confidence intervals (Cls) were estimated to assess these associations in seven studies that included 5151 cases and 1003 controls. In the overall analysis, the $309 \mathrm{G}$ allele was significantly associated with a decreased $\mathrm{PCa}$ risk $(\mathrm{OR}=0.85,95 \% \mathrm{Cl}: 0.74-0.97)$; this was also the case for the homozygous comparison $(\mathrm{OR}=0.72,95 \% \mathrm{Cl}: 0.55-0.95)$ and the dominant genetic model $(\mathrm{OR}=0.79,95 \% \mathrm{Cl}$ : $0.65-0.96)$. The $309 \mathrm{G}$ allele was also found to be significantly associated with lower degrees of $\mathrm{PCa}$ malignancy $(\mathrm{OR}=0.85,95 \% \mathrm{Cl}$ : $0.75-0.96)$ in the overall analysis, as well as in the heterozygous comparison ( $\mathrm{OR}=0.79,95 \% \mathrm{Cl}: 0.65-0.96)$, homozygous comparison ( $\mathrm{OR}=0.76,95 \% \mathrm{Cl}: 0.58-0.98)$ and dominant genetic model $(\mathrm{OR}=0.81,95 \% \mathrm{Cl}: 0.68-0.96)$. Furthermore, grouping analysis showed that the $309 \mathrm{G}$ allele in Caucasians was significantly correlated with a decreased $\mathrm{PCa}$ risk $(\mathrm{OR}=0.77,95 \% \mathrm{Cl}$ : $0.61-0.96)$; this was also the case in the homozygous comparison ( $\mathrm{OR}=0.51,95 \% \mathrm{Cl}: 0.31-0.86)$. The grouping analysis also showed that the 309G variant in Caucasians was significantly associated with a lower degree of PCa malignancy in all of the genetic models. In addition, we found that the $309 \mathrm{G}$ variant in Caucasians was significantly associated with a slower PCa clinical progression in all of the genetic models. In summary, our meta-analysis showed that the MDM2 309G variant was significantly associated with a decreased PCa risk, lower malignant degree and slower clinical progression in Caucasians, but there was no obvious association in the Asian population. Asian Journal of Andrology (2012) 14, 726-731; doi:10.1038/aja.2012.65; published online 20 August 2012

Keywords: clinical progression; malignant degree; MDM2; meta-analysis; polymorphism; prostate cancer; risk

\section{INTRODUCTION}

Prostate cancer $(\mathrm{PCa})$ is one of the most common solid tumours and is a common cause of death in men worldwide. However, the incidence of PCa has notable ethnic and geographic variations. ${ }^{1,2}$ There were an estimated 192280 newly diagnosed cases of PCa and 27360 deaths from PCa in the United States in 2008; in particular, the incidence in the Africa-American population was approximately 60 times that of the Han population in China. ${ }^{1-3}$ The aetiology of human PCa is complex and remains largely unknown. ${ }^{3,4}$

The human mouse double-minute 2 protein (MDM2) is an E3-ubiquitin ligase that binds, inhibits and promotes the degradation of the tumour suppressor protein $\mathrm{p} 53 .^{5}$ As a key negative regulator of $\mathrm{p} 53$, the overexpression of MDM2 is associated with accelerated tumour progression and the lack of response to therapy in various human malignancies. ${ }^{6-9}$ Recently, two polymorphic sites (T309G and C1797G) in the MDM2 promoter region have been shown to affect the affinity of MDM2 for binding to the stimulatory protein 1 and CAAT/enhancer binding protein $\alpha$ genes, respectively, which results in higher MDM2 expression levels. ${ }^{10,11}$
Although two polymorphic sites of the MDM2 gene (rs2279744 and rs937282) have been studied, only the rs2279744 (T309G) site has been reported frequently as a risk factor for PCa, with inconsistent results. $^{12,14,16-18}$ Moreover, some researchers have investigated the relationships between the T309G polymorphism and various clinical or pathological features of $\mathrm{PCa},{ }^{12-15,16-18}$ among which the malignant degree and clinical progression have been studied with the same stratified standards as each other. Therefore, we performed a meta-analysis of all eligible casecontrol studies and nested case-control studies to determine a more precise estimation of the PCa risk and clinical features (malignant degree and clinical progression) associated with the MDM2 T309G polymorphism.

\section{MATERIALS AND METHODS}

Publication search

We performed PubMed searches with the terms 'MDM2' or 'mouse double-minute 2', 'polymorphism' and 'prostate cancer' for articles published in English, and the last search update was performed on 29 March 2011. All eligible studies were reviewed, and their bibliographies

\footnotetext{
${ }^{1}$ Department of Urology, First Affiliated Hospital of Nanjing Medical University, Nanjing 210029, China and ${ }^{2}$ Cancer Biotherapy Centre, First Affiliated Hospital of Nanjing Medical University, Nanjing 210029, China

* The authors contributed equally to this work

Correspondence: Dr M Gu (gumin6663300@163.com)

Received: 31 March 2012; Revised: 17 May 2012; Accepted: 25 May 2012; Published online: 20 August 2012
} 
were also examined for other relevant publications. Relevant review articles were manually searched to find additional eligible studies. If more than one article had been published using the same series of study subjects, we only chose the most recent or most complete study for this meta-analysis.

\section{Inclusion and exclusion criteria}

The included articles met the following criteria: based on a casecontrol study or a nested case-control study; contained information on PCa risk or clinical features (malignant degree and clinical progression) associated with the MDM2 T309G polymorphism; and provided sufficient genotype frequencies for a meta-analysis. The major reasons for the exclusion of studies were as follows: no control population, insufficient available data and duplicate data.

\section{Data extraction}

All data were carefully extracted from the eligible publications independently by two co-authors (WG and PL), and any disagreements were resolved by discussion between the two authors. The collected data from each eligible article included the first author's last name, year of publication, country of origin, ethnicity, definitions of clinical features, characteristics of controls, numbers of genotyped cases and controls, numbers of genotyped cases grouped according to clinical features, source of cases (familial or sporadic), genotyping methods and quality control. Different ethnicity descents were categorized as Caucasian, Asian or African (Table 1).

\section{Statistical analysis}

The strength of association between the MDM2 T309G polymorphism and the risks or clinical features of PCa was measured by odds ratios (ORs) with 95\% confidence intervals (CIs). The statistical significance of the summary OR was determined using the $Z$-test. For exploring the associations between the MDM2 T309G polymorphism and PCa risk, we analysed the allelotype comparisons (309G vs. T), heterozygous comparisons (GT vs. TT), homozygous comparisons (GG vs. TT) and dominant genetic model comparisons (GG+GT vs. TT) between the cases and controls. To analyse the association of the T309G polymorphism and clinical features of PCa, we evaluated the same effects by stratified analyses according to the malignant degree and clinical progression of cases.

The heterogeneity assumption was verified by a $\chi^{2}$-based $Q$-test. If the $P$ value of the $Q$-test was more than 0.05 , which indicated a lack of heterogeneity among the studies, then the summary OR estimation for each study was calculated using the fixed-effects model (the
Mantel-Haenszel method). Otherwise, the random-effects model (the DerSimonian-Laird method) was used. ${ }^{19}$ We performed meta-regression analyses to explore the reasons of heterogeneity among these studies. Subgroup analyses were used to avoid the influence of heterogeneity among these studies, which were grouped by similar characteristics, such as ethnicity and source of cases. Inter-study variance $\left(I^{2}\right)$ was used to quantify the degree of heterogeneity, and the percentage of $I^{2}$ was used to describe the extent of heterogeneity explained by these characteristics. Potential publication bias was determined using Egger's linear regression test with a funnel plot. All statistical analyses were performed using the Stata software (version 11.0; StataCorp LP, College Station, TX, USA) and two-sided $P$ values. $P<0.05$ was considered statistically significant.

\section{RESULTS}

\section{Summary of included studies}

A total of seven studies that included 5151 cases and 1003 controls met the inclusion criteria. ${ }^{12-18}$ Of these studies, five studies (872 cases and 1003 controls) were used to analyse the association of the MDM2 T309G polymorphism and PCa risk. ${ }^{12,14,16-18}$ Five studies including 1485 cases of high-grade malignancy (Gleason score $\geqslant 7$ ) and 927 cases of lowgrade malignancy (Gleason score $<7$ ) were used to determine the association between the T309G polymorphism and the malignant degree. $^{12-15,17}$ Three studies including 697 advanced cases and 1403 localized cases were analysed to study the association between the T309G polymorphism and the clinical progression of cases. ${ }^{12,13,15}$ The characteristics of each study are summarized in Table 1. Five studies ${ }^{12,14,16-18}$ were included in the PCa risk analysis, and frequencymatched controls were used for age, sex and ethnicity. All seven of the studies focused on sporadic PCa (SPC) cases. All studies mentioned quality control methods for genotyping, such as randomly repeated assays or validation by directed sequencing. All studies stated that the distribution of genotypes in the control groups ${ }^{12,14,16-18}$ or case subjects ${ }^{13,15}$ was consistent with Hardy-Weinberg equilibrium (Table 1).

Jaboin et al. ${ }^{15}$ studied 206 SPC cases, including 98.06\% Caucasians and only $1.94 \%$ African-Americans; we classified this study by ethnicity as a study of Caucasians. After grouping by ethnicity, there were two studies ${ }^{17,18}$ of Caucasian descendants and three studies ${ }^{12,14,16}$ of Asian descendants for the PCa risk analysis associated with the MDM2 T309G polymorphism, three studies ${ }^{13,15,17}$ of Caucasian descendants and two studies ${ }^{12,14}$ of Asian descendants for the analysis of the malignant degree, and two studies ${ }^{13,15}$ of Caucasian descendants and one study $^{12}$ of Asian descendants for the analysis of the clinical progression (Tables 2 and 3).

Table 1 The main characteristics of the included studies

\begin{tabular}{|c|c|c|c|c|c|c|c|c|c|c|c|}
\hline First author & $\begin{array}{l}\text { Publication } \\
\text { year }\end{array}$ & $\begin{array}{l}\text { Publishing } \\
\text { country }\end{array}$ & $\begin{array}{l}\text { Subject } \\
\text { ethnicity }\end{array}$ & $\begin{array}{l}\text { Case } \\
\text { number }\end{array}$ & $\begin{array}{l}\text { Case } \\
\text { source }\end{array}$ & $\begin{array}{l}\text { Control } \\
\text { number }\end{array}$ & $\begin{array}{l}\text { HWE } \\
\text { test }\end{array}$ & $\begin{array}{l}\text { Genotyping } \\
\text { method }\end{array}$ & $\begin{array}{l}\text { Risk } \\
\text { analysis }\end{array}$ & $\begin{array}{l}\text { Malignant } \\
\text { degree } \\
\text { analysis }\end{array}$ & $\begin{array}{l}\text { Clinical } \\
\text { progression } \\
\text { analysis }\end{array}$ \\
\hline$X u^{12}$ & 2010 & China & Asian & 209 & SPC & 268 & Yes & PCR-RFLP & Yes & Yes & Yes \\
\hline $\operatorname{Sun}^{13}$ & 2010 & USA & Caucasian & 4073 & SPC & 0 & Yes & Mass spectrometry & NM & Yes & Yes \\
\hline Mandal $^{14}$ & 2010 & India & Asian & 192 & SPC & 224 & Yes & PCR-RFLP & Yes & Yes & NM \\
\hline Jaboin $^{15}$ & 2009 & USA & Caucasian $^{a}$ & 206 & SPC & 0 & Yes & Pyrosequencing & NM & Yes & Yes \\
\hline Hirata $^{16}$ & 2009 & Japan & Asian & 140 & SPC & 167 & Yes & PCR-RFLP & Yes & NM & NM \\
\hline Stoehr ${ }^{17}$ & 2008 & Germany & Caucasian & 145 & SPC & 124 & Yes & PCR-RFLP & Yes & Yes & NM \\
\hline Kibel $^{18}$ & 2008 & USA & Caucasian & 186 & SPC & 220 & Yes & Pyrosequencing & Yes & NM & NM \\
\hline
\end{tabular}

Abbreviations: HWE, Hardy-Weinberg equilibrium; NM, not mentioned; PCR, polymerase chain reaction; RFLP, restriction fragment length polymorphism; SPC, sporadic prostate cancer.

a $98 \%$ for Caucasians and $2 \%$ for African-Americans. 


\section{MDM2 T309G polymorphism and PCa risk}

No study was found that focused on the associations of the MDM2 T309G polymorphism and PCa risk in Africans; however, we observed a significant difference in the MDM2 309G allele frequency between Asian controls and Caucasian controls ( $48.9 \%$ vs. 38.4\%, $P<0.01$ ).

In the overall analysis, we found that the $309 \mathrm{G}$ allele was significantly associated with a decreased risk for PCa compared with the 309T allele ( $\mathrm{OR}=0.85,95 \% \mathrm{CI}: 0.74-0.97, P=0.170$ for heterogeneity); this was also the case for the homozygous comparison ( $\mathrm{OR}=0.72$, 95\% CI: $0.55-$ $0.95, P=0.130$ for heterogeneity) and the dominant genetic model ( $\mathrm{OR}=0.79,95 \%$ CI: $0.65-0.96, P=0.119$ for heterogeneity) (Table 2 and Figure 1a). There was no significant heterogeneity found in the overall analysis (Table 2); therefore, we did not assess the source of heterogeneity in any of the genetic models. In addition, the grouping analysis based on ethnicity showed that the 309G allele in Caucasians was significantly correlated to a decreased $\mathrm{PCa}$ risk $(\mathrm{OR}=0.77,95 \% \mathrm{CI}$ : $0.61-0.96, P=0.918$ for heterogeneity), and the same result was observed in the homozygous comparison ( $\mathrm{OR}=0.51,95 \% \mathrm{CI}$ : $0.31-$ 0.86, $P=0.458$ for heterogeneity) (Table 2 and Figure 1a). In Asians, there was no significant correlation between the T309G polymorphism and PCa risk; however, we found significant heterogeneity in the heterozygous comparison ( $P=0.041$ for heterogeneity) and dominant genetic model comparison ( $P=0.031$ for heterogeneity) (Table 2).

\section{MDM2 T309G polymorphism and the malignant degree of PCa cases}

To analyse the association between the MDM2 T309G polymorphism and the malignant degree of PCa, we defined low-grade malignancy as a Gleason score from 2 to $6(<7)$ and high-grade malignancy as a Gleason score from 7 to $10(\geqslant 7)$ for the meta-analysis. In the overall analysis, the $309 \mathrm{G}$ allele was found to be significantly associated with a lower malignant degree compared with the 309T allele $(\mathrm{OR}=0.85$, 95\% CI: $0.75-0.96, P=0.144$ for heterogeneity); this was also the case for the heterozygous comparison $(\mathrm{OR}=0.79,95 \% \mathrm{CI}$ : $0.65-0.96$, $P=0.381$ for heterogeneity), homozygous comparison $(\mathrm{OR}=0.76$, 95\% CI: $0.58-0.98, P=0.232$ for heterogeneity) and dominant genetic model $(\mathrm{OR}=0.81,95 \% \mathrm{CI}$ : $0.68-0.96, P=0.146$ for heterogeneity) (Table 3 and Figure 2a). Moreover, there was no significant heterogeneity found in the overall analysis (Table 3 ), and therefore, we did not assess the source of heterogeneity in the genetic models.

Furthermore, the grouping analysis based on ethnicity showed that the 309G allele in Caucasians was significantly correlated with a lower malignant degree $(\mathrm{OR}=0.82,95 \% \mathrm{CI}$ : $0.72-0.94, P=0.251$ for heterogeneity), and the same result was observed in the heterozygous comparison ( $\mathrm{OR}=0.78,95 \% \mathrm{CI}$ : $0.64-0.95, P=0.239$ for heterogeneity), homozygous comparison $(\mathrm{OR}=0.71,95 \% \mathrm{CI}$ : $0.54-0.93, P=0.392$ for heterogeneity) and dominant genetic model ( $\mathrm{OR}=0.76$, 95\% CI: $0.63-$ 0.92, $P=0.194$ for heterogeneity) (Table 3 and Figure 2a). In Asians, there was no significant correlation between the T309G polymorphism and PCa risk. The OR and 95\% CI were only reported by Mandal and Mittal, ${ }^{14}$ except for the dominant genetic model comparison, because the allelotype frequencies and partial genotype frequencies were not available in the study by $\mathrm{Xu}$ et al. ${ }^{12}$ (Table 3 ).

\section{MDM2 T309G polymorphism and the clinical progression of $\mathrm{PCa}$ cases}

For the association between the MDM2 T309G polymorphism and clinical progression of $\mathrm{PCa}$, we defined localized $\mathrm{PCa}$ as clinical stages of $\mathrm{T}_{1-2} \mathrm{~N}_{0} \mathrm{M}_{0}$ and advanced PCa as clinical stages of $\mathrm{T}_{3-4} \mathrm{~N}_{\mathrm{x}} \mathrm{M}_{\mathrm{x}}$, $T_{x} N_{1} M_{x}$ or $T_{x} N_{x} M_{1}$. Because the allelotype frequencies and partial genotype frequencies were not available in the study by $\mathrm{Xu}$ et al., ${ }^{12}$ we only used the OR and 95\% CI of the dominant genetic model comparison in the overall analysis, which was not ideal; however, it had significant heterogeneity ( $P=0.026$ for heterogeneity) (Table 3 and Figure 3a). Therefore, we assessed the source of heterogeneity in the dominant genetic model by publication year (2009 or 2010), ethnicity (Caucasian or Asian) and sample size (less than or more than subjects 200 in both localized cases and advanced cases). As a result, publication year $(P=0.924)$, ethnicity $(P=0.353)$ and sample size $(P=0.236)$ did not account for the substantial heterogeneity.

Furthermore, we performed subgroup analyses by ethnicity, which showed positive results in all of the genetic models. We found that the 309G allele was significantly associated with a lower clinical progression of PCa in Caucasians compared with the 309T allele $(\mathrm{OR}=0.83$, 95\% CI: $0.72-0.96, P=0.127$ for heterogeneity); this was also the case for the heterozygote comparison $(\mathrm{OR}=0.79,95 \% \mathrm{CI}$ : $0.64-0.97$, $P=0.266$ for heterogeneity), homozygous comparison $(\mathrm{OR}=0.72,95 \%$ CI: $0.53-0.98, P=0.169$ for heterogeneity) and dominant genetic model $(\mathrm{OR}=0.77,95 \% \mathrm{CI}: 0.63-0.94, P=0.174$ for heterogeneity) (Table 3 and Figure 3a).

\section{Sensitivity analyses}

In the dominant genetic model comparison for the association between MDM2 T309G variants and PCa risk, sensitivity analyses indicated that the positive summary OR and 95\% CI became negative when any of the three independent studies ${ }^{14,16,17}$ were omitted (Figure 1b). Similarly, sensitivity analyses also showed that one independent study by Sun et al. ${ }^{13}$ effectively influenced the positive summary OR and 95\% CI for the PCa malignant degree associated with the T309G polymorphism in the dominant genetic model (Figure 2b). In addition, sensitivity analyses indicated that the pooled negative OR and 95\% CI for PCa clinical progression were not influenced significantly, which indicated that the meta-analysis results were stable in the dominant genetic model (Figure 3b).

Table 2 Stratified analyses of the MDM2 T309G polymorphism on PCa risk

\begin{tabular}{|c|c|c|c|c|c|c|c|c|c|c|}
\hline \multirow{2}{*}{ Ethnicities } & \multirow{2}{*}{ N } & \multirow{2}{*}{ Cases/controls } & \multicolumn{2}{|c|}{ Gallele vs. $T$ allele } & \multicolumn{2}{|l|}{ GT vs. $T T$} & \multicolumn{2}{|l|}{ GG vs. $T T$} & \multicolumn{2}{|l|}{ GT+GG vs. $T T$} \\
\hline & & & OR $(95 \% \mathrm{Cl})$ & $P^{a}$ & OR $(95 \% \mathrm{Cl})$ & $\mathrm{P}^{a}$ & OR $(95 \% \mathrm{Cl})$ & $\mathrm{P}^{a}$ & OR $(95 \% \mathrm{Cl})$ & $P^{a}$ \\
\hline Caucasian & 2 & $331 / 344$ & $0.77(0.61-0.96)$ & 0.918 & $0.84(0.61-1.16)$ & 0.353 & $0.51(0.31-0.86)$ & 0.458 & $0.76(0.56-1.04)$ & 0.558 \\
\hline Asian & 3 & $541 / 659$ & $0.90(0.76-1.05)$ & 0.075 & $0.80(0.49-1.30)^{\mathrm{b}}$ & 0.041 & $0.83(0.60-1.13)$ & 0.117 & $0.81(0.50-1.30)^{\mathrm{b}}$ & 0.031 \\
\hline
\end{tabular}

Abbreviations: $\mathrm{Cl}$, confidence interval; MDM2, mouse double-minute 2 protein; $N$, number of studies; OR, odds ratio; PCa, prostate cancer.

${ }^{a} P$ value of $Q$-test for heterogeneity test.

${ }^{\mathrm{b}} \mathrm{A}$ random-effect model was used when the $P$ value for the heterogeneity test was $<0.05$. 


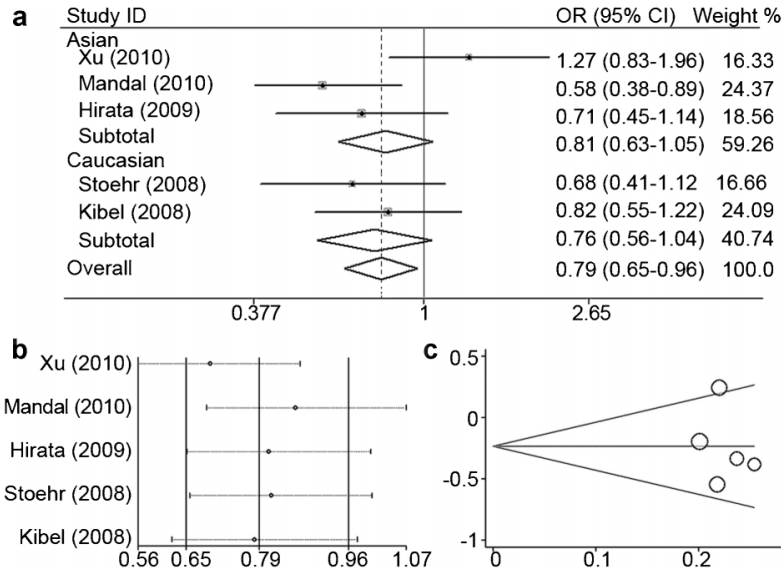

Figure 1 The forest plot, sensitivity analysis diagram and Begg's funnel plot for the PCa risk associated with the MDM2 T309G polymorphism in the dominant genetic model. (a) The forest plot of the fixed-effects model for PCa risk. The squares and horizontal lines correspond to the study-specific OR and $95 \% \mathrm{Cl}$, respectively. The area of the squares reflects the weight. The diamonds represent the summary ORs and $95 \% \mathrm{Cl}$. (b) The sensitivity analysis diagram for each study used to calculate the PCa risk. The given named-study is omitted. (c) Begg's funnel plot of the publication bias test. Each point represents a separate study for the $\mathrm{PCa}$ risk. $\mathrm{Cl}$, confidence interval; $\mathrm{OR}$, odds ratio; $\mathrm{PCa}$, prostate cancer.

\section{Publication bias}

We adopted Begg's funnel plot and Egger's test to assess the publication bias. The shape of the funnel plots seemed symmetrical and did not suggest the presence of a publication bias in either the dominant genetic model for PCa risk (Figure 1c) or clinical progression (Figure 3c). Egger's test was used to provide statistical evidence for funnel plot symmetry. As expected, the results did not show any obvious evidence of a publication bias in either the dominant genetic models for PCa risk $(P=0.775)$ or clinical progression $(P=0.148)$. However, in the dominant genetic models for PCa malignant degree, we found an obvious publication bias using Egger's test $(P=0.038)$ (Figure 2c). When the study by Sun et al. ${ }^{13}$ was excluded, the publication bias for malignant degree was effectively removed $(P=0.596)$.

\section{DISCUSSION}

Only a small percentage of cancers, approximately $5 \%-10 \%$, are entirely hereditary. ${ }^{20-22}$ Like most cancers, prostate carcinogenesis is a complex process involving a multifactorial interplay between genetic and environmental factors, and as a result, the effect of a single polymorphism is unlikely to be substantial. A study of a polymorphism may be of limited value in predicting the risk and clinical features of PCa. ${ }^{1,3,5}$ Therefore, to explore the association between MDM2 T309G variants and the risk or clinical features, we performed a meta-analysis of seven published studies. To our knowledge, this is the first metaanalysis to explore the association of the T309G polymorphism with the risk, malignant degree and clinical progression of $\mathrm{PCa}$.

The MDM2 T309G polymorphism is a plausible carcinogenic hereditary factor owing to the important role of MDM2 in the cellular p53 pathway, and it has been found to be correlated with cancer risks in other organ sites. ${ }^{23}$ The MDM2 309G variant raises the affinity of transcription factors, such as Sp1, which results in increased MDM2 synthesis. ${ }^{7}$ Moreover, some studies have proven that MDM2 overexpression is related to gene amplification in several malignancies. ${ }^{24,25}$

In our study, the overall analysis indicated that the 309G variant was significantly associated with a decreased PCa risk without any

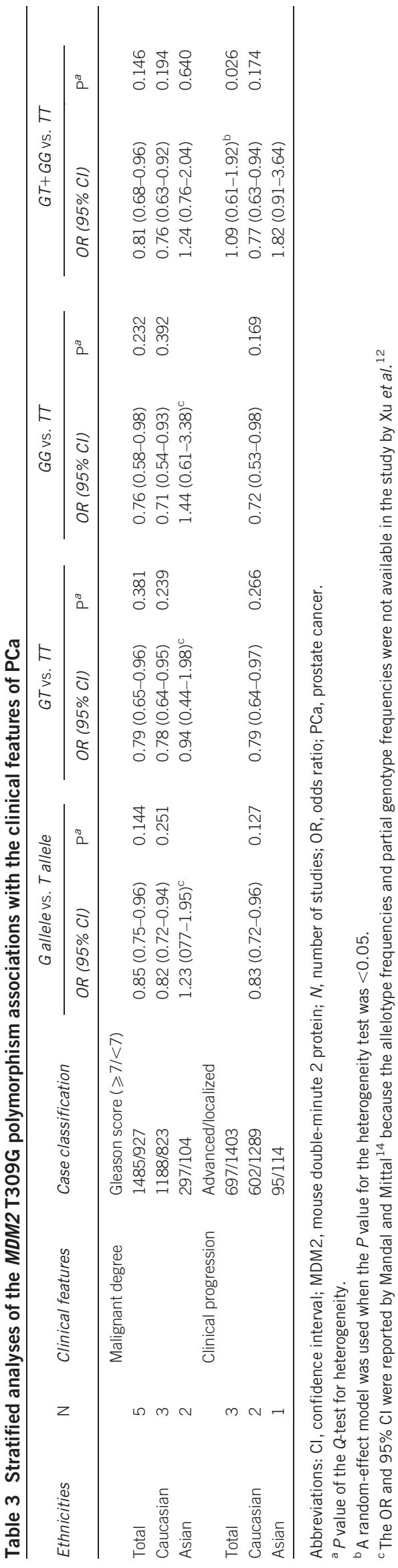




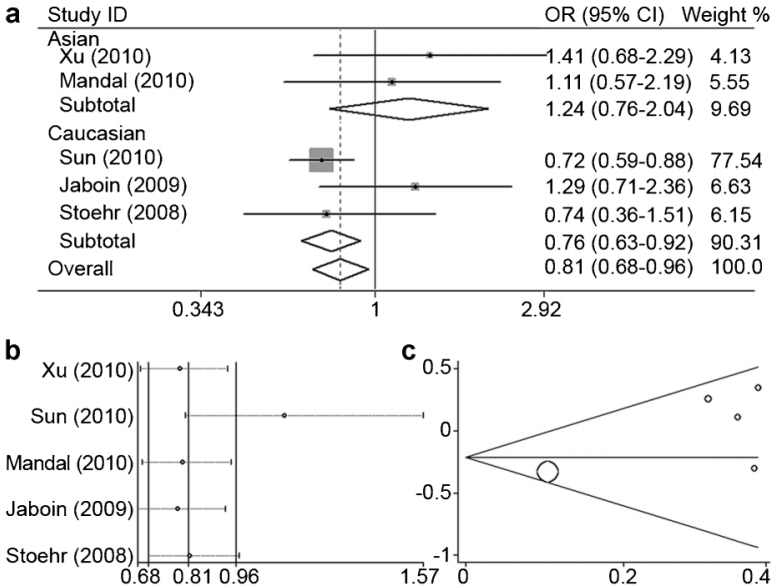

Figure 2 The forest plot, sensitivity analysis diagram and Begg's funnel plot for the malignant degree of PCa cases associated with the MDM2 T309G polymorphism in the dominant genetic model. (a) The forest plot of the fixed-effects model for the malignant degree of PCa cases. The squares and horizontal lines correspond to the study-specific OR and $95 \% \mathrm{Cl}$, respectively. The area of the squares reflects the weight. The diamonds represent the summary ORs and $95 \%$ $\mathrm{Cl}$. (b) The sensitivity analysis diagram of each study used to determine for the malignant degree of PCa cases. The given named-study is omitted. (c) Begg's funnel plot of the publication bias test. Each point represents a separate study for the malignant degree of $\mathrm{PCa}$ cases. $\mathrm{Cl}$, confidence interval; MDM2, mouse double-minute 2 protein; OR, odds ratio; $\mathrm{PCa}$, prostate cancer.

significant heterogeneity in the allelotype comparison, homozygous comparison or dominant genetic model (Table 2). After grouping by ethnicity, we found different results for Caucasians and Asians: the 309G allele in allelotype comparison and the GG genotype in the homozygous comparison were significantly correlated with a decreased PCa risk in Caucasians but not in Asians (Table 2). We believe that these inconsistent results could be explained by the following points. First, the discrepancy of potential distribution frequencies of alleles among different ethnicities may be the cause of the inconsistency because we found that the frequency of the 309G allele was significantly lower in Caucasian controls than in Asian controls $(P<0.01)$, which is consistent with the much higher incidence of PCa in Caucasians than in Asians. ${ }^{1,4,5}$ Second, the mechanism of PCa development is dependent on the interactions of genetic factors and environmental agents. ${ }^{20-22,26}$ The environmental factors and life habits are very different for individual races. ${ }^{27}$ Other factors, such as time-lag bias and publication bias, may also have a role, but we examined these possibilities and found no significant effects on the PCa risk.

To analyse the relationship between the T309G polymorphism and the clinical features of PCa, we studied the malignant degree and clinical progression of $\mathrm{PCa}$, the two most important aspects in tumour biological behaviour. In the overall analysis, the MDM2 309G allele was found to be significantly associated with a lower PCa malignant degree in the allelotype comparison as well as in the GT genotype in the heterozygous comparison, the GG genotype in the homozygous comparison and the GT+GG genotypes in the dominant genetic model (Table 3). In contrast, we did not find a positive result for PCa clinical progression in dominant genetic model in the overall analysis (Table $\mathbf{3}$ and Figure 3a). After grouping by ethnicity, the subgroup analyses showed that the $309 \mathrm{G}$ variant was significantly associated with both a lower malignant degree and slower clinical progression in all of the genetic models in Caucasians; however, we did not find these associations in Asians (Table 3). Previous studies

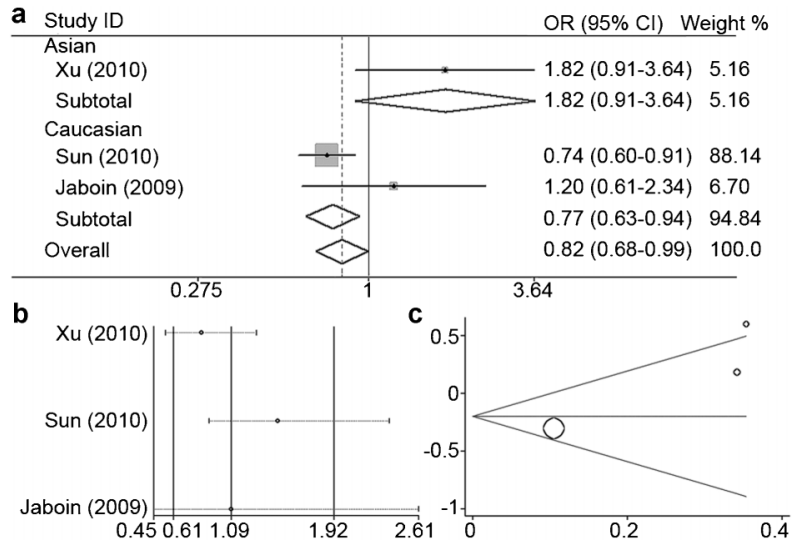

Figure 3 The forest plot, sensitivity analysis diagram and Begg's funnel plot for the clinical progression of PCa cases associated with the MDM2 T309G polymorphism in the dominant genetic model. (a) The forest plot of the fixed-effects model for the clinical progression of PCa cases. The squares and horizontal lines correspond to the study-specific OR and $95 \% \mathrm{Cl}$, respectively. The area of the squares reflects the weight. The diamonds represent the summary ORs and $95 \%$ $\mathrm{Cl}$. (b) The sensitivity analysis diagram of each study used to determine the clinical progression of PCa cases. The given named-study is omitted. (c) Begg's funnel plot of publication bias test. Each point represents a separate study for the clinical progression of PCa cases. $\mathrm{Cl}$, confidence interval; MDM2, mouse double-minute 2 protein; OR, odds ratio; PCa, prostate cancer.

have implicated the MDM2 309G allele as a high-risk allele in many other carcinomas, ${ }^{28-31}$ but our data showed that the MDM2 309G allele was significantly associated with a decreased risk for PCa and a lower malignant degree and slower clinical progression of PCa. According to these conflicting results for different tumours, we presume that each variant of the T309G polymorphic site may be associated with an increased risk for different types of cancers. Furthermore, the increased cancer risk could be associated with a specific interaction between a person's genetic background and environmental factors. ${ }^{26}$ For some cancers, such as PCa and breast cancer, hormones may be an important environmental factor that influences the effects of genetic variance because many studies have indicated that prostate and breast cancer are dependent on sexual hormones to grow and progress in the early stage. ${ }^{32}$ Bond et al. ${ }^{33}$ reported that the MDM2 T309G polymorphism can accelerate tumour formation in a gender-specific and hormone-dependent manner. Recently, the MDM2 309G variant has been shown to be bound more efficiently by the transcriptional factor $\mathrm{Sp} 1$ (a co-activator for many hormone receptors) than the $309 \mathrm{~T}$ allele, ${ }^{10,11}$ and the oestrogen receptor also binds the promoter of MDM2 in the region of SNP309. ${ }^{33} \mathrm{Hu}$ et al. ${ }^{34}$ have found that oestrogen preferentially induces the transcription of MDM2 from the SNP309 promoter in oestrogen-responsive cells, and the level of MDM2 in SNP 309GG cells is higher than in heterozygous GT or TT cells. Bond et al. ${ }^{33}$ hypothesized that women with a $\mathrm{G}$ allele would preferentially benefit from lower oestrogen levels, which could slow the progression of some cancers. To date, no evidence on androgen signalling has been presented. Considering the importance of the androgen axis in prostate tumorigenesis and the results of our metaanalyses, we believe that the effect of MDM2 T309G variants may also be dependent on hormonal signalling. Further studies focused on the androgen levels associated with T309G variants are needed.

Some limitations of this meta-analysis should be acknowledged. First of all, there were no studies focused on Africans in this metaanalysis, which hindered the comprehensive investigation of the associations between the MDM2 T309G polymorphism and the risk and 
clinical features of PCa. Second, the sample sizes were dissimilar among the studies, and therefore, the statistical power of each study was also very different. Because of the large sample size of $4073 \mathrm{PCa}$ cases in the Caucasian study by Sun et al..$^{13}$ the statistical power of the results in Asians was weaker for the malignant degree and clinical progression of PCa. In addition, the sensitivity analyses showed that the study by Sun et al. ${ }^{13}$ effectively influenced the positive summary OR and 95\% CI of PCa malignant degree associated with the T309G polymorphism in the dominant genetic model (Figure 2b). Therefore, further studies with larger sample sizes of the Asian population are needed to confirm these conflicting results between ethnicities. Third, only published studies were included in the meta-analysis, which could explain the obvious publication bias for the PCa malignant degree caused by the Sun et al.'s study ${ }^{13}$ (Figure 2c).

Our meta-analysis also had some advantages. Firstly, the risk, malignant degree and clinical progression of PCa were all studied in the meta-analysis. Secondly, the quality of studies included in the meta-analysis was satisfactory and strictly met the inclusion criteria. Thirdly, all seven studies focused on SPC cases, mentioned quality control methods for genotyping and stated that the distribution of genotypes in control groups ${ }^{12,14,16-18}$ or case subjects ${ }^{13,15}$ was consistent with the Hardy-Weinberg equilibrium (Table 1), which all increased the statistical power of the meta-analysis.

\section{CONCLUSIONS}

This meta-analysis showed that the MDM2 309G variant is significantly associated with a decreased PCa risk, lower malignant degree and clinical progression in Caucasians, but there was no obvious association found in Asians. Considering the limitations of the present meta-analysis, further research with larger sample sizes and different ethnicities is needed to confirm these results. Moreover, other possible confounding factors, such as environmental and lifestyle factors, should also be controlled and considered when assessing the effect of inherited factors on PCa tumorigenesis and progression.

\section{AUTHOR CONTRIBUTIONS}

JY, MG and WG conceived and designed the study. WW, JXZ and PL collected the data. NHS, JY and WG performed statistical analyses. JY and LXH drafted and revised the manuscript. All authors read and approved the final version.

\section{COMPETING FINANCIAL INTERESTS}

The authors declare that they have no competing financial interests.

1 Bono AV. The global state of prostate cancer: epidemiology and screening in the second millennium. BJU Int 2004; 94 (Suppl 3)1-2.

2 Zhang L, Yang BX, Zhang HT, Wang JG, Wang HL et al. Prostate cancer: an emerging threat to the health of aging men in Asia. Asian J Androl 2011; 13: 574-8.

3 Yang J, Xu DL, Lu Q, Han ZJ, Tao J et al. Prostate cancer risk and aggressiveness associated with the CYP1B1 4326C/G (Leu432Val) polymorphism: a meta-analysis of 2788 cases and 2968 controls. Asian J Androl 2012, 14: 560-5.

4 Jemal A, Siegel R, Ward E, Hao Y, Xu J et al. Cancer statistics, 2009. CA Cancer J Clin 2009; 59: 225-49.

5 Schaid DJ. The complex genetic epidemiology of prostate cancer. Hum Mol Genet 2004; 13 (Sepc No. 1): R103-21.
6 Haupt Y, Maya R, Kazaz A, Oren M. Mdm2 promotes the rapid degradation of p53. Nature 1997: 387: 296-9.

7 Hu W, Feng Z, Ma L, Wagner J, Rice JJ et al. A single nucleotide polymorphism in the MDM2 gene disrupts the oscillation of p53 and MDM2 levels in cells. Cancer Res 2007; 67: 2757-65.

8 Khor LY, Desilvio M, Al-Saleem T, Hammond ME, Grignon DJ et al. MDM2 as a predictor of prostate carcinoma outcome: an analysis of Radiation Therapy Oncology Group Protocol 8610. Cancer 2005; 104: 962-7.

9 Nayak MS, Yang JM, Hait WN. Effect of a single nucleotide polymorphism in the murine double minute 2 promoter (SNP309) on the sensitivity to topoisomerase IItargeting drugs. Cancer Res 2007; 67: 5831-9.

10 Bond GL, Hu W, Levine A. A single nucleotide polymorphism in the MDM2 gene: from a molecular and cellular explanation to clinical effect. Cancer Res 2005; 65: 5481-4.

11 Wang M, Zhang Z, Zhu H, Fu G, Wang S et al. A novel functional polymorphism C1797G in the MDM2 promoter is associated with risk of bladder cancer in a Chinese population. Clin Cancer Res 2008; 14: 3633-40.

12 Xu B, Xu Z, Cheng G, Min ZC, Mi Y et al. Association between polymorphisms of TP53 and MDM2 and prostate cancer risk in southern Chinese. Cancer Genet Cytogenet 2010; 202: 76-81.

13 Sun T, Lee GS, Oh WK, Pomerantz M, Yang M et al. Single-nucleotide polymorphisms in p53 pathway and aggressiveness of prostate cancer in a Caucasian population. Clin Cancer Res 2010; 16: 5244-51

14 Mandal RK, Mittal RD. Are cell cycle and apoptosis genes associated with prostate cancer risk in North Indian population? Urol Oncol. 2010 Sep 3. [Epub ahead of print]

15 Jaboin JJ, Hwang M, Perez CA, Cooper C, Chen $\mathrm{H}$ et al. No evidence for association of the MDM2-309 T/G promoter polymorphism with prostate cancer outcomes. Urol Oncol 2011; 29: 319-23.

16 Hirata H, Hinoda Y, Kikuno N, Suehiro Y, Shahryari V et al. Bcl2-938C/A polymorphism carries increased risk of biochemical recurrence after radical prostatectomy. J Urol 2009; 181: 1907-12.

17 Stoehr R, Hitzenbichler F, Kneitz B, Hammerschmied CG, Burger M et al. Mdm2 SNP309 polymorphism in prostate cancer: no evidence for association with increased risk or histopathological tumour characteristics. Br J Cancer 2008; 99: 78-82.

$18 \mathrm{Kibel}$ AS, Jin CH, Klim A, Luly J, A Roehl K et al. Association between polymorphisms in cell cycle genes and advanced prostate carcinoma. Prostate 2008; 68: 1179-86.

19 DerSimonian R, Laird N. Meta-analysis in clinical trials. Control Clin Trials 1986; 7 177-88.

20 Taby R, Issa JP. Cancer epigenetics. CA Cancer J Clin 2010; 60: 376-92.

21 Frank SA. Inheritance of cancer. Discov Med 2004; 4: 396-400.

22 Hemminki K, Li X, Czene K. Familial risk of cancer: data for clinical counseling and cancer genetics. Int J Cancer 2004; 108: 109-14.

$23 \mathrm{Hu} Z$, Jin G, Wang L, Chen F, Wang X et al. MDM2 promoter polymorphism SNP309 contributes to tumor susceptibility: evidence from 21 case-control studies. Cance Epidemiol Biomarkers Prev 2007; 16: 2717-23.

24 Knappskog S, Lønning PE. MDM2 promoter SNP285 and SNP309; phylogeny and impact on cancer risk. Oncotarget 2011; 2: 251-8.

25 Slack A, Shohet JM. MDM2 as a critical effector of the MYCN oncogene in tumorigenesis. Cell Cycle 2005; 4: 857-60.

26 Pfeifer GP. Environmental exposures and mutational patterns of cancer genomes. Genome Med 2010; 2: 54.

27 Sharad S, Srivastava A, Ravulapalli S, Parker P, Chen Y et al. Prostate cancer gene expression signature of patients with high body mass index. Prostate Cancer Prostatic Dis 2011; 14: 22-9.

28 Terry K, McGrath M, Lee IM, Buring J, de Vivo I. MDM2 SNP309 is associated with endometrial cancer risk. Cancer Epidemiol Biomarkers Prev 2008; 17: 983-6.

29 Bond GL, Hu W, Bond EE, Robins H, Lutzker SG et al. A single nucleotide polymorphism in the MDM2 promoter attenuates the p53 tumor suppressor pathway and accelerates tumor formation in humans. Cell 2004;119: 591-602.

30 Economopoulos KP, Sergentanis TN. Differential effects of MDM2 SNP309 polymorphism on breast cancer risk along with race: a meta-analysis. Breast Cancer Res Treat 2010; 120: 211-6.

31 Bai J, Dai J, Yu H, Shen H, Chen F. Cigarette smoking, MDM2 SNP309, geneenvironment interactions, and lung cancer risk: a meta-analysis. J Toxicol Environ Health A 2009; 72: 677-82.

32 Cunha GR, Ricke W, Thomson A, Marker PC, Risbridger G et al. Hormonal, cellular, and molecular regulation of normal and neoplastic prostatic development. J Steroid Biochem Mol Biol 2004; 92: 221-36.

33 Bond GL, Hirshfield KM, Kirchhoff T, Alexe G, Bond EE et al. MDM2 SNP309 accelerates tumor formation in a gender-specific and hormone-dependent manner. Cancer Res 2006; 66: 5104-10.

34 Hu W, Feng Z, Ma L, Wagner J, Rice JJ et al. A single nucleotide polymorphism in the MDM2 gene disrupts the oscillation of p53 and MDM2 levels in cells. Cancer Res 2007; 67: 2757-65. 